\title{
Magnetic Resonance Angiographic Evaluation of Anatomic Variations of the Circle of Willis
}

\author{
Fethi Emre Ustabaşıoğlu, (i) Serdar Solak \\ Department of Radiology, Trakya University, Edirne, Turkey
}

\begin{abstract}
Introduction: The Circle of Willis, an anastomotic vascular network at the base of the brain, forms an important collateral pathway to protect cerebral blood perfusion. This study aims to investigate the different anatomic variations of the Willis circle and their prevalence.

Methods: The patient population for this single-centre, retrospective study consisted of patients who had brain magnetic resonance angiography. A total of 580 subjects were included in this study. The three-dimensional time-of-flight technique was used for MRA imaging to evaluate the anatomy of the circle of Willis.

Results: The findings showed that the anterior part of the circle of Willis had a complete structure in $82 \%$ of the cases. The vascular structure of the posterior part was complete in $22.2 \%$ of the cases.

Discussion and Conclusion: Posterior segment variations are more frequent in the Circle of Willis evaluation with magnetic resonance angiography, and the most common type of variation in our study was bilateral posterior communicating artery absence.

Keywords: Circle of Willis; magnetic resonance angiography; variation.
\end{abstract}

$\mathrm{T}$ he Willis polygon (WP) at the base of the brain is a vital collateral system to maintain cerebral blood flow in the event of a decrease in blood flow that may occur in one or more of the proximal vessels ${ }^{[1]}$.

In the occlusion of major cerebral arteries, WP plays a role in the correction of decreased brain perfusion by providing vascularization of the stenotic area from the contralateral carotid artery and basilar artery [2] .

The Willis polygon, first defined by Thomas Willis in 1664, is formed by the merging of the branches of the internal carotid arteries (ICA) and the basilar artery ${ }^{[3]}$. The anterior cerebral artery (ASA) originating from the ICA and the anterior communicating artery (AkomA) connecting both ASAs constitute the anterior segment of the polygon; the posterior segment comprises the posterior communicating arter- ies (Pcoma) and the posterior cerebral arteries (PCA) (Fig. 1). WP shows several variations, and studies have concluded that variations play an important role in patients with carotid artery disease ${ }^{[4-7]}$.

Due to the rapid advances in imaging techniques, WP, which was previously evaluated with angiography, can now be easily evaluated with magnetic resonance angiography (MRA), which is a cheaper and non-invasive method. Another advantage of this method is that it can be carried out without the need for a contrast agent. The three-dimensional images required to evaluate WP are prepared by the "time of flight"'(3D TOF) method.

In this study, we aimed to investigate the frequency and distribution of WP variations by gender.

Correspondence (iletişim): Fethi Emre Ustabaşıŏlu, M.D. Trakya Universitesi Tip Fakultesi Hastanesi Radyoloji Anabilim Dali, Edirne, Turkey Phone (Telefon): +90 5325707267 E-mail (E-posta): ustabasioglu@hotmail.com 


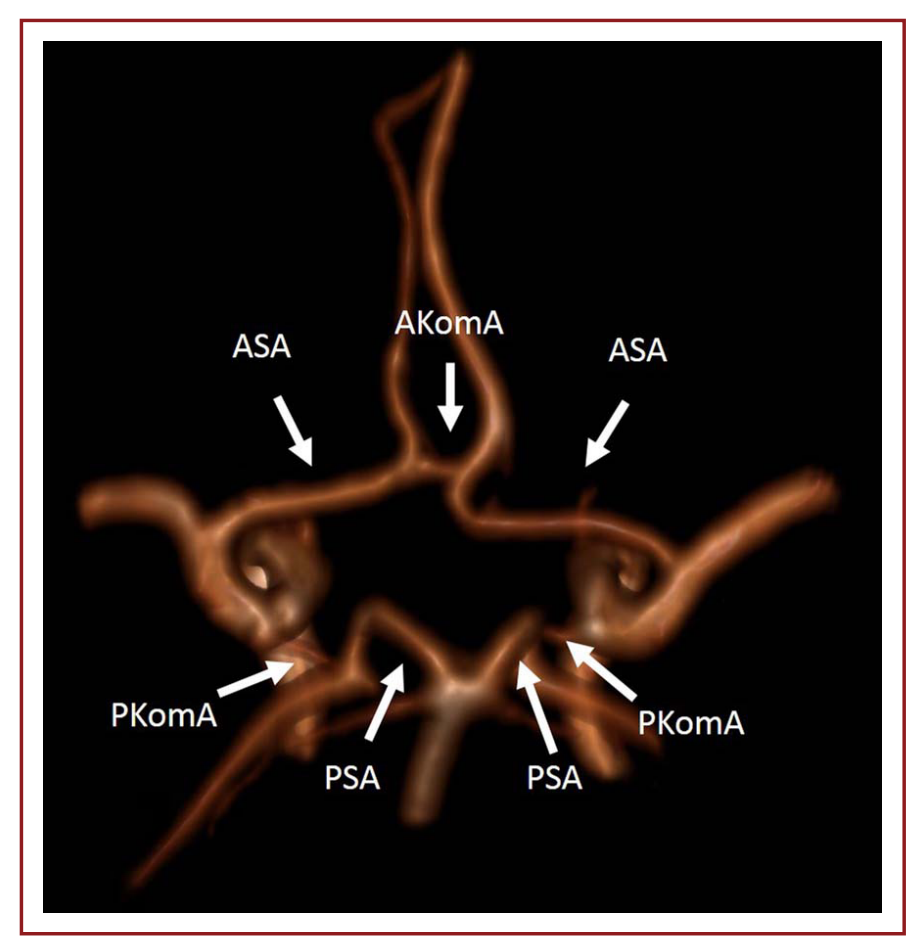

Figure 1. 3-D TOF MRA image of the vessels forming the Willis polygon. ACA: Anterior cerebral artery. ACOMA: Anterior communicating artery. PCA: Posterior cerebral artery. PComa: The posterior communicating artery.

\section{Materials and Methods}

\section{Selection and Description of Cases}

Ethics Committee approval was given to our study by Trakya University School of Medicine Ethics Committee. Between January 2014 and February 2018, the images of 580 patients who had undergone cranial magnetic resonance an- giography (MRA) with the 3D time of flight technique (3D TOF) were examined retrospectively. Thirty-three patients who could not obtain sufficient diagnostic quality images due to intensive motion artefacts were excluded from this study. Variations of the Willis polygons were classified according to the criteria defined in Figure 2 and Figure 3.

\section{Technical Data}

Examinations of all patients were performed using the eight-channel cranial coil in 1.5T MRI and employing 3D TOF MRA technique. TR and TE parameters were set to 24 and 7 milliseconds ( $\mathrm{ms}$ ) and flip angle to 25 degrees. The imaging area -Field of View- was selected as 230×150 mm and the section thickness as $0.6 \mathrm{~mm}$.

The TOF MRA technique uses sequences called gradient sequences that allow rapid data collection. This technique is mainly used to evaluate the flow changes emerging in the direction of the longitudinal magnetization vector in vascular structures. In this study, the vessels were examined axially and the flow was perpendicular to the crosssectional plane. The obtained data were constructed in 3D, and images were created with high spatial analysis. Raw images were processed using a maximum intensity projection (MIP) algorithm to obtain angiographic images.

\section{Results}

The mean age of the 580 patients consisting of 252 (43.4\%) female and $328(56.6 \%)$ male patients was $53.28 \pm 16.87$ years. Anatomic variations of WP were examined into anterior and posterior sections ${ }^{[8]}$. Figures 4 and 5 show anterior and posterior variations of WP.

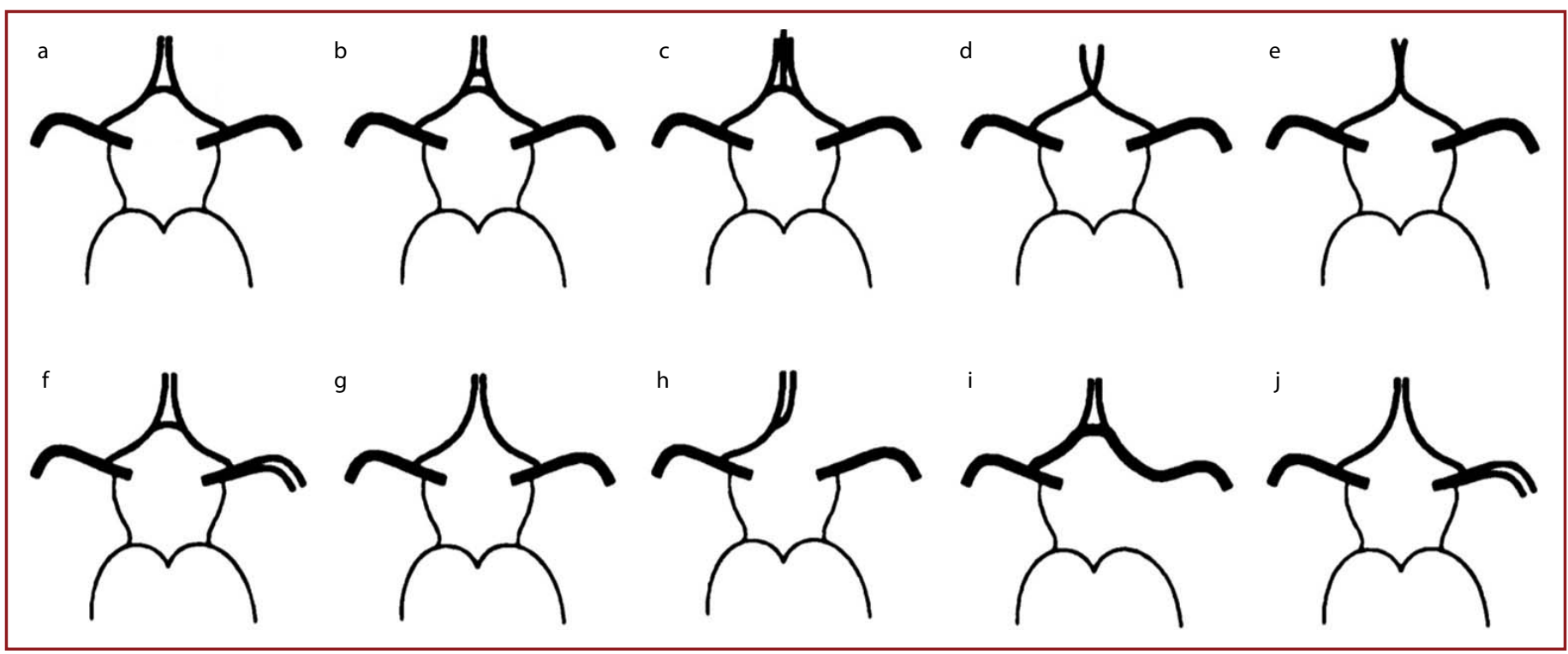

Figure 2. Anatomical variations of the anterior part of the Willis polygon. 


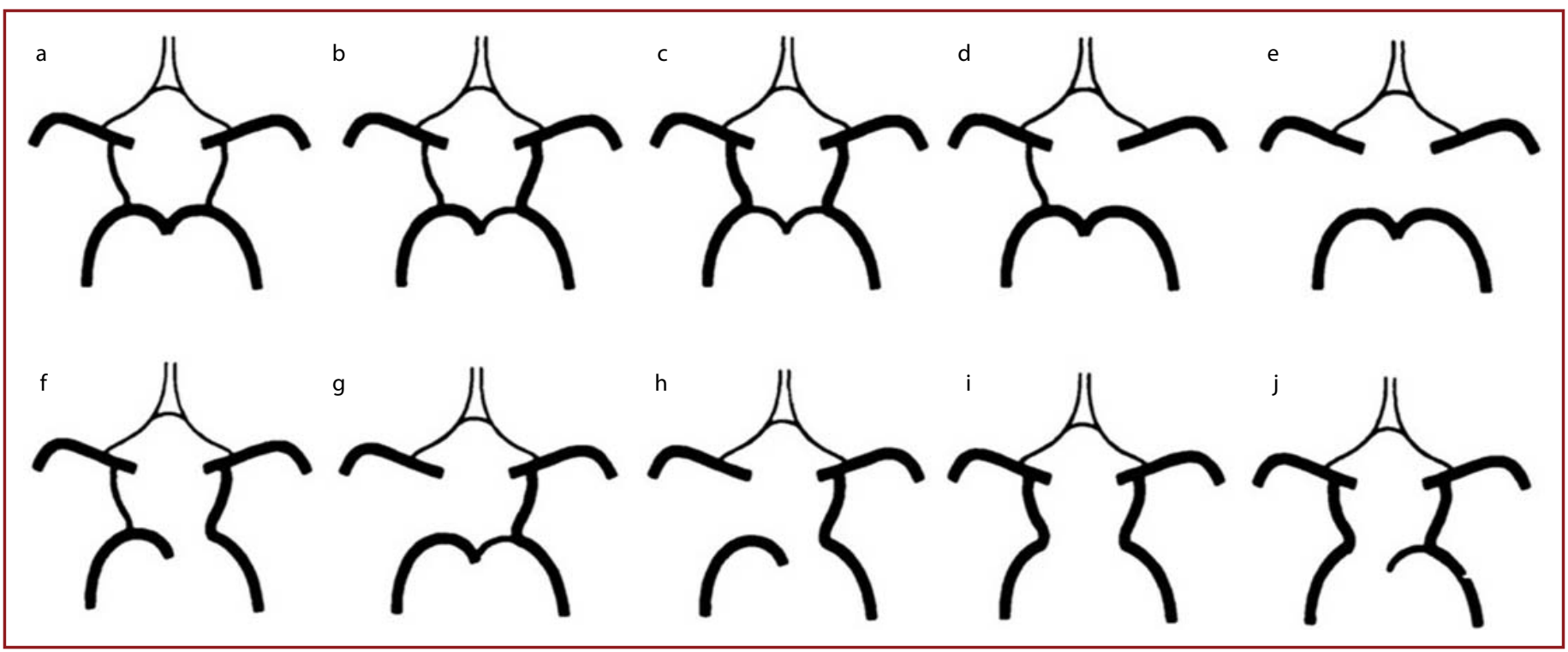

Figure 3. Anatomical variations of the posterior part of the Willis polygon.

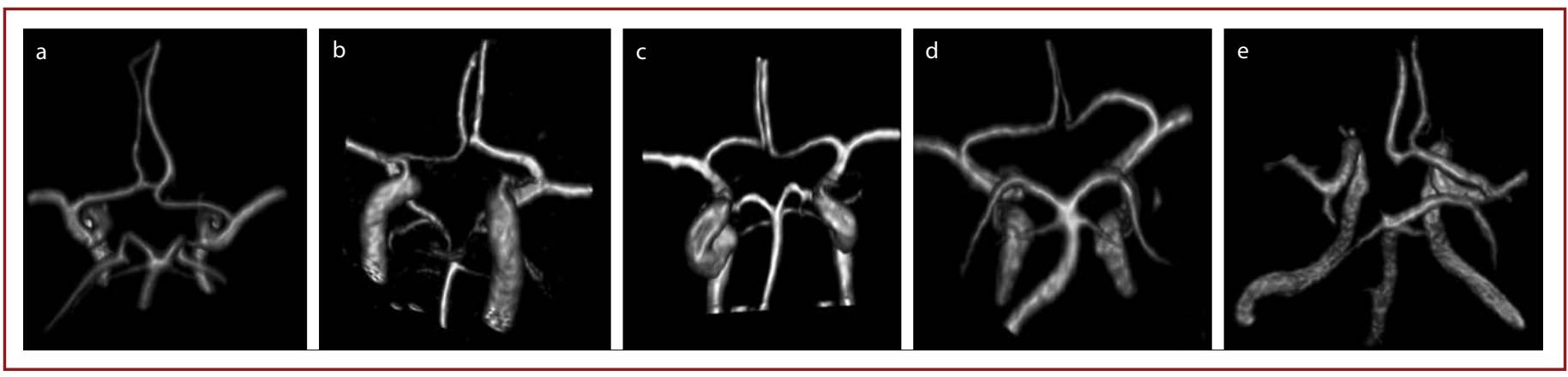

Figure 4. (a-e) 3D colour reconstructions of TOF MRA images of the anatomical variations of the anterior part of the Willis polygon, (a) Type a anatomical variation. (b) Type d anatomical variation. (c) Type e anatomical variation. (d) Type g anatomical variation. (e) Type h anatomical variation.

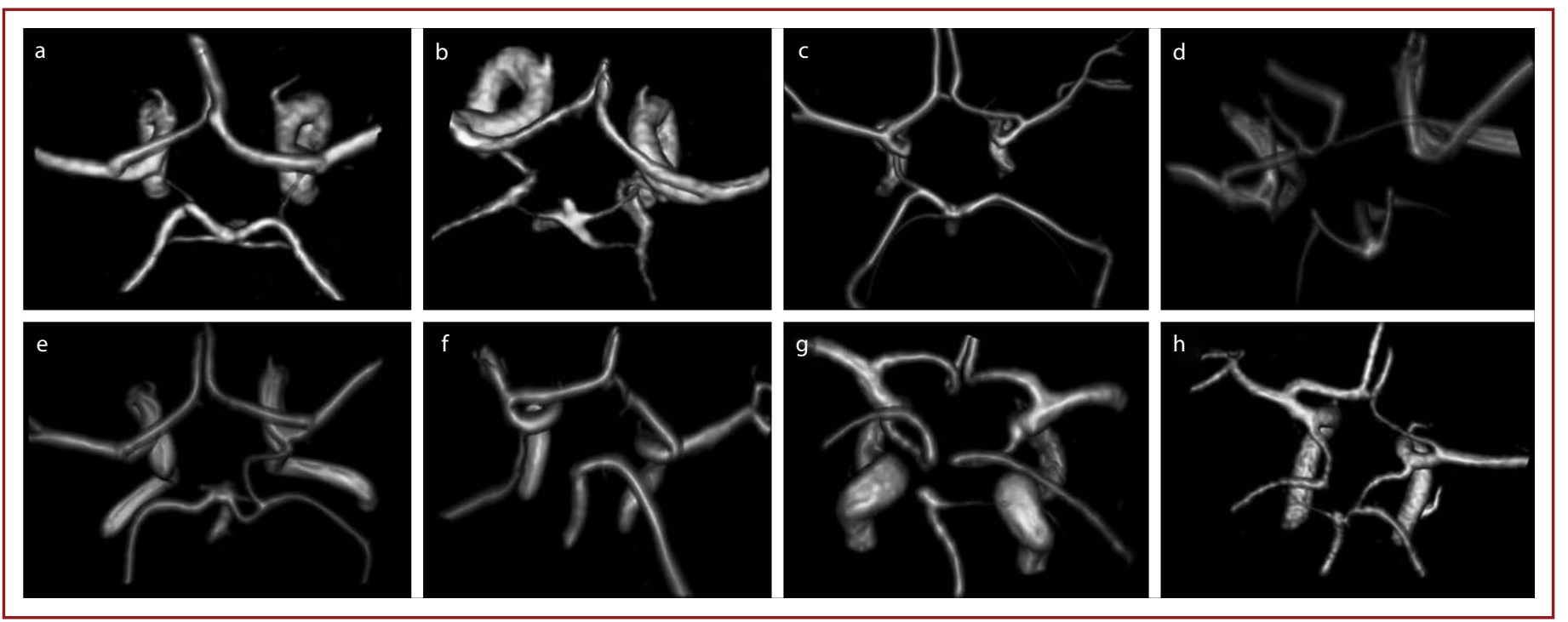

Figure 5. (a-h) 3D colour reconstructions of TOF MRA images of the anatomical variations of the posterior portion of the Willis polygon, (a) Type a anatomical variation. (b) Type c anatomical variation. (c) Type d anatomical variation. (d) Type e anatomical variation. (e) Type g anatomical variation. (f) Type $h$ anatomical variation. (g) Type i anatomical variation. (h) Type j anatomical variation. 
Our findings showed that the anterior part of the Willis polygon was in normal configuration (type a) in 421 (72.6\%) cases. In 110 (18.8\%) cases, posterior circulation was in normal configuration (type a). The most common variant configuration $(14.2 \%)$ of the anterior part of WP was type $g$ variation where hypoplastic and aplastic anterior communicating artery (AcomA) was observed. The complete anterior part of WP was seen $82 \%$ (476 patients) of the patients. The most common variant configuration in the posterior section was type e configuration seen in 254 patients (43.6\%). Complete posterior WP was seen in $22.2 \%$ (129 patients) of the patients.

\section{Discussion}

WP is a vascular network that connects both sides of the brain's anterior circulation to each other and the vertebrobasilar system. In the embryological period, primitive ICAs are divided into cranial and caudal sections. The cranial portion terminates as a primitive olfactory artery and then forms the ASA. AcomA occurs when the plexiform vascular network between the two ASAs joins at the midline.

Caudal ICA sections are regressed by making anastomosis with the dorsal longitudinal neural arteries and form the recommunicating segments of Pcoma and PCA [9]. However, the presence of complete anterior and posterior WP is an uncommon condition and was found to be only $21 \%$ in macroscopic autopsy studies ${ }^{[7]}$.

WP provides maintenance of perfusion pressure, which is critical to brain function, especially in the event of occlusion of proximal cerebrovascular structures.

WP anomaly and its variations and hemodynamic stress factors associated with these variations are important because they predispose to aneurysm formation. Studies have shown that the frequency of WP asymmetry or anomaly is higher in patients with an aneurysm [10].

In a study, the findings indicated that stroke risk is increased in patients with ICA occlusion with hypoplasia or absence of PcomA [11].

TOF MRA is preferred because it does not require contrast material and does not contain ionizing radiation, and it can show aneurysms up to $5 \mathrm{~mm}$ in the diagnosis of intracranial aneurysms at a high rate ${ }^{[12,13]}$.

In their study, Ghazali et al. ${ }^{[14]}$ compared three-dimensional TOF MRA and digital subtraction angiography to evaluate the morphology of WP arteries. Our findings showed that MRA is a more sensitive method for the evaluation of other WP arteries, except PComA.
In our series, the most common type of anterior configuration was WP anterior part was found to be complete in $82 \%$ of the cases. These results are consistent with similar studies in the literature ${ }^{[15-18]}$. In our study, the most common anterior configuration type was complete arterial circulation anterior circulation (type a) associated with single AcomA in $72.6 \%$ of the patients which is in parallel with similar studies in the literature ${ }^{[15-17]}$. In addition, the second most common anterior variation was found to be aplasia-hypoplasia of AcomA (type g) in $14.2 \%$ of the patients which was in accordance with the studies in the literature ${ }^{[16]}$.

In $22.2 \%$ of the cases, the WP posterior section was observed to be complete, and our results were similar when compared with similar studies in the literature ${ }^{[15-17]}$. The most common posterior variation (type e) was characterized by the absence of both PComAs and was observed in $43.6 \%$ of the patients. Our results were comparable to findings observed by Chen et al. and Kondori et al. in $42.8 \%$ and $44.7 \%$ of their patients, respectively. However, Naveen et al. observed this variation in a higher proportion (32.6\%) of patients compared to our study ${ }^{[15-17]}$. The second most common posterior variation of WP is the native type (type a) characterized by the presence of both PcomAs that was found in $18.8 \%$ of the cases. This result of ours was also evaluated to be in accordance with the findings obtained by Chen et al. ${ }^{[15]}$ and Kondori et al.'s ${ }^{[16]}$ studies. The fetal type PCA of WP was found in $21.6 \%$ of the cases in our study. In $18.7 \%$ of the cases, unilateral fetal type PCA (type $b, f, g, h$ ); in $2.9 \%$ of the cases, bilateral fetal type PCA (type $c, i$, j) were found. In our study, the rate of both-sided fetal type PCA was found to be low compared to the literature, and the rate of unilateral fetal type PCA was consistent with the literature $[15,17]$.

The most important limitation of our study is that the 3D TOF MRA technique decreases its visibility in vessels that are under a certain calibration or whose flow velocity is outside the prescribed velocity limits in this sequence, leading to incorrect evaluations.

\section{Conclusion}

In conclusion, posterior segment variations were more common in WP evaluation using MRA and, and the most common type of variation in our study was the bilateral absence of PcomA. PcomA absence or hypoplasia may increase the risk of stroke in patients with critical ICA stenosis or obstruction. Therefore, it is important to be aware of the variation in the absence of bilateral Pcoma in this patient group. 
Ethics Committee Approval: Trakya University Medical Faculty Dean Scientific Research Ethics Committee, TÜTF-BAEK 2018/112.

Peer-review: Externally peer-reviewed.

Authorship Contributions: Concept: F.E.U., S.S.; Design: F.E.U., S.S.; Data Collection or Processing: F.E.U., S.S.; Analysis or Interpretation: F.E.U., S.S.; Literature Search: F.E.U., S.S.; Writing: F.E.U., S.S.

Conflict of Interest: None declared.

Financial Disclosure: The authors declared that this study received no financial support.

\section{References}

1. Symonds C. The circle of Willis. Br Med J 1955;1:119-24.

2. Yeniceri IO, Cullu N, Deveer M, Yeniceri EN. Circle of Willis variations and artery diameter measurements in the Turkish population. Folia Morphol (Warsz) 2017;76:420-25.

3. Ustun C. NEUROwords Dr. Thomas Willis' famous eponym: the circle of Willis. J Hist Neurosci 2005;14:16-21.

4. Schomer DF, Marks MP, Steinberg GK, Johnstone IM, Boothroyd $\mathrm{DB}$, Ross MR, et al. The anatomy of the posterior communicating artery as a risk factor for ischemic cerebral infarction. $\mathrm{N}$ Engl J Med 1994;330:1565-70.

5. Miralles M, Dolz JL, Cotillas J, Aldoma J, Santiso MA, Giménez $A$, et al. The role of the circle of Willis in carotid occlusion: assessment with phase contrast MR angiography and transcranial duplex. Eur J Vasc Endovasc Surg 1995;10:424-30.

6. Battacharji SK, Hutchinson EC, Mccall AJ. The circle of Willis the incidence of developmental abnormalities in normal and infarcted brains. Brain 1967;90:747-58.

7. Riggs $H E$, Rupp C. Variation in form of circle of Willis: the relation of the variations to collateral circulation: anatomic analysis. Arch Neurol 1963;8:8-14.

8. Krabbe-Hartkamp MJ, Van der Grond J, De Leeuw FE, De Groot JC, Algra A, Hillen B, et al. Circle of Willis: morphologic varia- tion on three-dimensional time-of-flight MR angiograms. Radiology 1998:207:103-11.

9. Osborn AG. Diagnostic Cerebral Angiography. 2nd ed. Salt Lake City; 1999:105-16.

10. Alpers BJ, Berry RG. Circle of Willis in cerebral vascular disorders: the anatomical structure. Arch Neurol 1963;8:398-402.

11. Chuang YM, Liu CY, Pan PJ, Lin CP. Posterior communicating artery hypoplasia as a risk factor for acute ischemic stroke in the absence of carotid artery occlusion. J Clin Neurosci 2008;15:1376-81.

12. Deutschmann HA, Augustin M, Simbrunner J, Unger B, Schoellnast $H$, Fritz GA, et al. Diagnostic accuracy of 3D time-of-flight MR angiography compared with digital subtraction angiography for follow-up of coiled intracranial aneurysms: influence of aneurysm size. AJNR Am J Neuroradiol 2007;28:628-34.

13. Slater LA, Soufan C, Holt M, Chong W. Effect of flow diversion with silk on aneurysm size: a single center experience. Interv Neuroradiol 2015; 21:12-8.

14. Ghazali RM, Shuaib IL. Comparison between 3D TOF magnetic resonance angiography and intraarterial digital subtraction angiography in imaging the circle of Willis. Malays J Med Sci 2003;10:37-42.

15. Chen HW, Yen PS, Lee CC, Chen CC, Chang PY, Lee SK, et al. Magnetic resonance angiographic evaluation of circle of Willis in general population: a morphologic study in 507 cases. Chin J Radiol 2004; 29:223-29.

16. Jalali Kondori B, Azemati F, Dadseresht S. Magnetic Resonance Angiographic Study of Anatomic Variations of the Circle of Willis in a Population in Tehran. Arch Iran Med 2017;20:235-39.

17. Naveen SR, Bhat V, Karthik GA. Magnetic resonance angiographic evaluation of circle of Willis: A morphologic study in a tertiary hospital set up. Ann Indian Acad Neurol 2015;18:391-7.

18. Hartkamp MJ, Van der Grond J. Investigation of the circle of Willis using MR angiography. Medicamundi 2000;44:20-7. 